\title{
ESTUDO DA INFLUÊNCIA DA LIOFILIZAÇÃO NOS PARÂMETROS DE COR DE FRUTAS TROPICAIS
}

\author{
H. LUNARDI ${ }^{1 *}$, G.S. ROSA ${ }^{1}$ \\ ${ }^{1}$ Universidade Federal do Pampa, Curso de Engenharia Química \\ *e-mail: heloise.lunardi@gmail.com
}

\begin{abstract}
RESUMO
A comercialização mundial de produtos a partir de frutas vem crescendo nos últimos anos. Dentre estes, as frutas desidratadas têm ganhado espaço, visto que possuem algumas vantagens, como uma maior vida de prateleira. A liofilização é uma das técnicas de secagem de alimentos, que consiste na retirada da água do material através da sublimação, garantindo assim que as propriedades organolépticas da amostra praticamente não se alterem. Uma das características fundamentais para um alimento, natural ou processado, é a sua cor. Esta é proveniente dos pigmentos presentes nos alimentos, destacando-se a clorofila, as antocianinas e os carotenoides. O objetivo deste trabalho foi estudar a influência da liofilização em parâmetros de cor (luminosidade, intensidade de verde/vermelho e intensidade do amarelo/azul), assim como na umidade de frutas tropicais. Foram utilizadas amostras de abacaxi, banana e manga nas formas in natura e liofilizadas. No geral os resultados obtidos de luminosidade, intensidade de vermelho e de amarelo foram maiores nas amostras secas do que nas in natura. Ainda, verificou-se que em todas as amostras há a predominância da cor amarela sobre a vermelha. Quanto a umidade observaram-se baixos valores para as amostras liofilizadas (10 a $16 \%$, b.u.).
\end{abstract}

\section{INTRODUÇÃO}

A comercialização mundial de produtos provenientes de frutas vem apresentando significativo aumento nos últimos anos. Neste cenário, o Brasil destaca-se entre os países com maior produção de frutas tropicais do mundo (FAO, 2003).

Entre os produtos processados, as frutas desidratadas destacam-se pelo baixo custo de transporte, fácil obtenção e estabilidade à temperatura ambiente. Além disto, devido à dificuldade do desenvolvimento de microorganismos, estas frutas apresentam maior vida de prateleira (MUJUMDAR, 2004).
A liofilização é um dos métodos existentes para a secagem de alimentos. Esta consiste na retirada da água do material por meio da sublimação da parte congelada em temperaturas baixas e sob vácuo (IBARZ e BARBOSA-CANOVAS, 1999).

A grande vantagem da liofilização é que devido ao processamento ser a baixas temperaturas e com ausência de ar atmosférico, as propriedades químicas e organolépticas da amostra praticamente não sofrem alteração (GAVA, 1994).

Segundo Bobbio e Bobbio (1992), um dos parâmetros de extrema importância para a aceitabilidade de um alimento é a sua 


\section{ENEMP \\ ENE \\ DE SISTEMAS PARTICULADOS \\ São Carlos - SP}

aparência, deste modo a cor do produto tornase uma característica fundamental, seja em produtos naturais ou processados.

As cores nos alimentos de origem vegetal são provenientes dos pigmentos presentes, dentre estes os principais são a clorofila, os carotenoides e as antocianinas. As antocianinas normalmente proporcionam tons de vermelho, púrpura ou violeta, enquanto que os carotenoides são responsáveis pelas cores entre o amarelo e o laranja, presentes na maioria das frutas (BOBBIO e BOBBIO, 1992).

Para a detecção destes pigmentos ou de alterações dos mesmos, usualmente são empregados equipamentos capazes de realizar a medição da cor de alimentos. Deste modo, a análise de cor torna-se uma opção mais simples e rápida de estimar compostos coloridos presentes em alimentos do que a análise química (FRANCIS, 1983).

Este trabalho teve como objetivo liofilizar amostras de abacaxi, banana e manga, e analisar a influência desse processo na cor e no conteúdo de umidade das amostras. Ainda, objetivou-se a comparação destes resultados com amostras comerciais liofilizadas.

\section{MATERIAIS E MÉTODOS}

Para este estudo foram utilizadas como amostras abacaxi, banana e manga in natura, assim como amostras comerciais liofilizadas (marca Jasmine) destes mesmos frutos.

Inicialmente foi realizado o preparo das amostras in natura. Este consistiu na lavagem, retirada da casca e corte das frutas em pequenos pedaços. As amostras utilizadas estão apresentadas na Figura 1.
Figura 1 - Amostras in natura e comerciais.

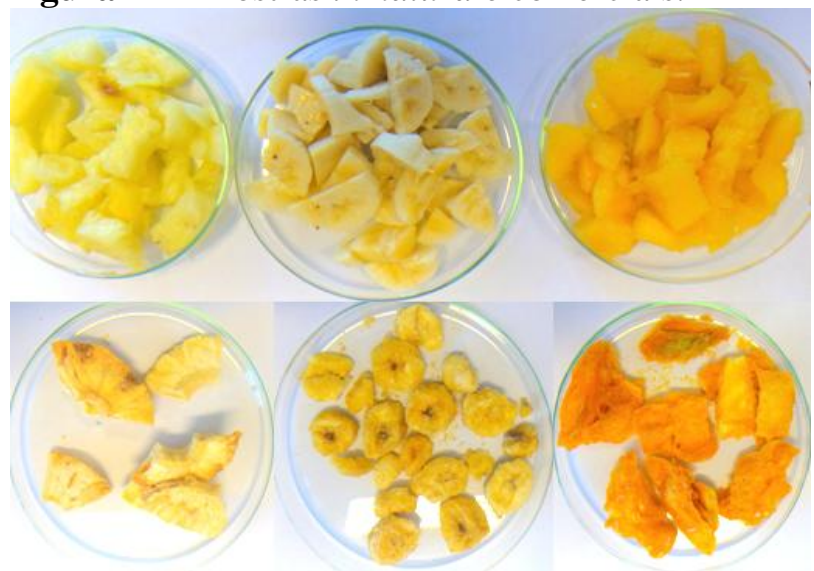

Fonte: Autor (2015).

Para realizar o processo de liofilização as amostras in natura foram congeladas previamente em um freezer por $24 \mathrm{~h}$, a uma temperatura de $-18{ }^{\circ} \mathrm{C}$. Após o congelamento, as amostras foram inseridas em um liofilizador (LS3000, Terroni), apresentado na Figura 2.

Figura 2 - Liofilizador: (1) painel de controle; (2) câmara de secagem; (3) bomba de vácuo.

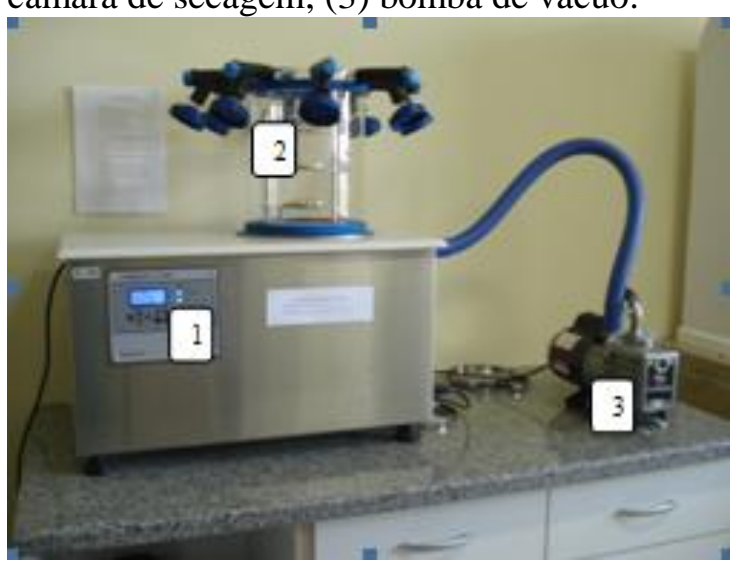

Fonte:Autor(2015).

O processo de liofilização foi realizado por $48 \mathrm{~h}$, sob vácuo e temperatura de $-50{ }^{\circ} \mathrm{C}$.

A avaliação da umidade das amostras in natura, seca comercial e liofilizadas neste trabalho foi feita através do método de secagem em estufa a $105{ }^{\circ} \mathrm{C}$ por $24 \mathrm{~h}$.

Para a análise dos parâmetros de cor das amostras foi utilizado um 
espectrofotômetro portátil (CM-2600D, Konica Minolta). Foram realizadas 5 repetições para cada amostra, observando os parâmetros de luminosidade $\left(\mathrm{L}^{*}\right)$, que varia de zero a 100 (preto/branco); intensidade de vermelho/verde $\mathrm{a}^{*}(+/-)$ e intensidade de amarelo/azul b* $(+/-)$.

A morfologia das amostras secas foi obtida utilizando um microscópio estereoscópio binocular (SMZ500, Motic).

Para avaliar os resultados obtidos das análises de cor foi realizado o teste de Tukey, ao nível de 5\% de significância, utilizando um software estatístico. Este teste permite realizar comparações múltiplas e determinar estatisticamente se há diferença significativa entre os resultados.

\section{RESULTADOS E DISCUSSÃO}

Os resultados do conteúdo de umidade (base úmida) das amostras in natura e secas estão expostos na Tabela 1.

Tabela 1 - Umidade (\% b.u.) das amostras.

\begin{tabular}{|c|c|c|c|}
\hline & $\mathbf{U}_{\text {abacaxi }}$ & $\mathbf{U}_{\text {banana }}$ & $\mathbf{U}_{\text {manga }}$ \\
\hline in natura & $86,8 \pm 0,1$ & $71,8 \pm 0,1$ & $84,8 \pm 1,2$ \\
\hline $\begin{array}{c}\text { Liof. } \\
48 \mathrm{~h}\end{array}$ & $16,3 \pm 0,3$ & $10,2 \pm 0,1$ & $8,8 \pm 0,2$ \\
\hline $\begin{array}{l}\text { Liof. } \\
\text { Comercial }\end{array}$ & $10,8 \pm 0,5$ & $10,5 \pm 0,5$ & $10,4 \pm 0,4$ \\
\hline
\end{tabular}

Fonte: Autor (2015)

Através da análise da Tabela 1 pode-se observar que os resultados encontrados para as amostras in natura foram muito próximos ao que a literatura reporta. Ramos et al. (2008) verificaram um valor de $86,5 \%$ de umidade para o abacaxi, enquanto Lima et al. (2012) reportaram 71,7 \% para a banana, e Moreira et al.(2013) 85,5\% para a manga.

Observa-se que o conteúdo de umidade presente nas amostras liofilizadas é muito menor do que nas amostras in natura. Isto já era esperado, visto que, o objetivo do processo de liofilização é a redução da umidade presente na amostra.

As amostras secas comerciais e obtidas neste trabalho, apresentaram valores de acordo com os parâmetros estabelecidos pela legislação para frutas secas. A Resolução CNNPA (Comissão Nacional de Normas e Padrões para Alimentos) no 12 de 1978 da Agência Nacional de Vigilância Sanitária caracteriza como fruta seca produtos com umidade inferior a $25 \%$, sendo este um parâmetro de proteção contra o desenvolvimento de micro-organismos deteriorantes e patogênicos (ANVISA, 1978).

As micrografias das amostras secas, com magnificação de $64 \mathrm{X}$, são mostradas na Figura 3.

Figura 3 - Micrografias com aumento de 64x de amostras de: (1) abacaxi, (2) banana e (3) manga liofilizadas; (4) abacaxi, (5) banana e (6) manga secas comerciais.

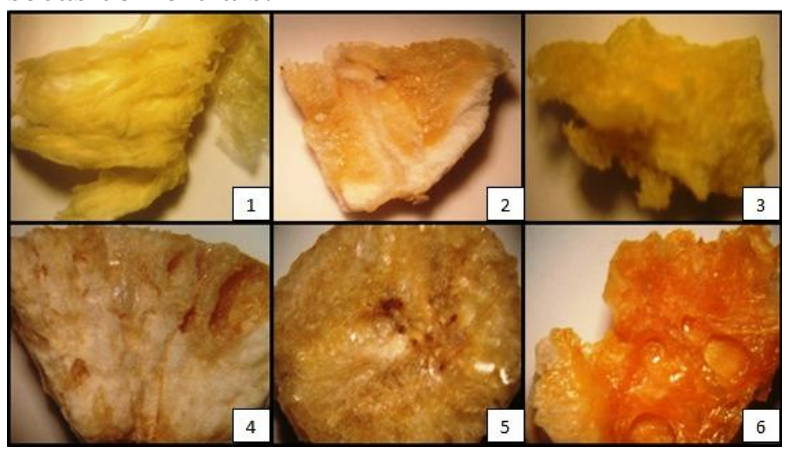

Fonte: Autor (2015).

Analisando as micrografias da superfície das amostras secas, verifica-se que a operação de liofilização proporcionou a secagem das amostras sem ocasionar grandes alterações em suas estruturas. Segundo Zotarelli et al (2012) isto ocorre pois a liofilização tende a preservar as características estruturais do produto.

A Tabela 2 apresenta os valores obtidos para os parâmetros de cor das amostras de abacaxi (L*, a*, b*).

Tabela 2 - Parâmetros de cor das amostra de abacaxi. 


\begin{tabular}{cccc}
\hline & $\mathbf{L}^{*}$ & $\mathbf{a}^{*}$ & $\mathbf{b}^{*}$ \\
\hline in natura & $66,2^{\mathrm{a}} \pm 2,8$ & $-0,9^{\mathrm{b}} \pm 0,2$ & $30,3^{\mathrm{c}} \pm 2,8$
\end{tabular}

Liof.

$48 \mathrm{~h}$

$$
70,4^{\mathrm{a}} \pm 4,4 \quad 0,3^{\mathrm{b}} \pm 1,1 \quad 34,2^{\mathrm{c}} \pm 5,5
$$

Liof.

Comercial

$$
76^{\mathrm{a}} \pm 7,5 \quad 4,3^{\mathrm{c}} \pm 1,6 \quad 31,2^{\mathrm{c}} \pm 2,5
$$

Obs.: médias seguidas pela mesma letra nas colunas não diferem estatisticamente ao nível de 5\% de significância pelo teste de Tukey.

Fonte: Autor (2015).

Analisando a Tabela 2, observa-se que em todas as amostras de abacaxi a cor amarela predomina sobre a cor vermelha. Ramos et al. (2008) reportaram valores de luminosidade de 62,1 ; de intensidade de vermelho de $-1,5$ e 22,6 para a intensidade de amarelo para amostras de abacaxi in natura, valores esses muito próximos aos encontrados neste estudo. Além disto, nota-se uma pequena intensidade de verde na amostra in natura, indicado pelo valor de $a^{*}$ negativo.

No caso de amostras secas em secador com fluxo de ar aquecido, Ramos et al. (2008) obtiveram valores de 3,5 e 31,9 para a intensidade de vermelho e de amarelo, respectivamente, valores estes que condizem com os resultados encontrados para a amostra comercial. Já as amostras liofilizadas apresentaram menor aumento na intensidade de vermelho do que a reportada por Ramos, indicando que esta técnica é eficaz na prevenção de reações de escurecimento, como já exposto por Krokida et al., (2000).

Em relação a luminosidade, Ramos et al., (2008) reportaram valor de 51,5 para o abacaxi seco, valor bem inferior ao obtido neste estudo. Esta discrepância pode ser explicada pelo método de secagem empregado por este autor.

Krokida et al., (2000) estudaram o efeito do método de secagem sobre parâmetros de cor. Estes observaram que a luminosidade diminuiu significativamente após a secagem convencional, enquanto que na liofilização este valor sofreu pequeno aumento. Deste modo, verificaram uma maior estabilidade dos parâmetros colorimétricos da amostra liofilizada.

Além disto, observa-se que para todas as amostras, os parâmetros de luminosidade e intensidade de amarelo não diferem estatisticamente entre si. Isto já não foi observado na intensidade de vermelho, a qual apresentou diferença estatística entre a amostra comercial e as demais.

Os resultados dos parâmetros de cor das amostras de banana são mostrados na Tabela 3.

Tabela 3 - Parâmetros de cor das amostras de banana.

\begin{tabular}{cccc}
\hline & $\mathbf{L}^{*}$ & $\mathbf{a}^{*}$ & $\mathbf{b}^{*}$ \\
\hline in natura & $72,4^{\mathrm{a}} \pm 5,2$ & $4,8^{\mathrm{b}} \pm 0,5$ & $26,8^{\mathrm{c}} \pm 3,5$ \\
Liof. & & & \\
$48 \mathrm{~h}$ & $72,5^{\mathrm{a}} \pm 3,1$ & $5,3^{\mathrm{b}} \pm 1,2$ & $30,2^{\mathrm{c}} \pm 2,4$ \\
Liof. & & & \\
Comercial & $71,5^{\mathrm{a}} \pm 2$ & $5,9^{\mathrm{b}} \pm 0,5$ & $39,5^{\mathrm{b}} \pm 0,9$
\end{tabular}

Obs.: médias seguidas pela mesma letra nas colunas não diferem estatisticamente ao nível de 5\% de significância pelo teste de Tukey.

Fonte: Autor (2015).

Através da Tabela 3, nota-se a proximidade dos valores de luminosidade obtidos. Ainda, é possível observar que a tonalidade amarela prevalece sobre a vermelha neste fruto independente do tratamento da amostra.

Os valores referentes aos parâmetros de cor das amostras de banana in natura estão próximos daqueles descritos por Oliveira (2007), que foram de 53,3 a 72 para a luminosidade, de 2,7 a 8 para a intensidade de vermelho e 20,3 a 29,7 para a intensidade de amarelo. Porém, as faixas dos parâmetros para a banana seca em secador com fluxo de ar aquecido, indicadas por este autor diferem dos obtidos neste trabalho. Oliveira (2007) reportou valores de luminosidade entre 58,3 e 
68,3; de contribuição de vermelho de 6,4 a 9,1 e de contribuição de amarelo de 18,8 a 22,2.

Assim, foi possível observar que a banana liofilizada apresentou menor variação nos valores de $a^{*}$ e $b^{*}$ do que na secagem convectiva. Indicando assim, que a liofilização é mais eficaz na prevenção da deterioração da cor dos produtos do que os demais métodos. Através da análise estatística observou-se que somente a cor da amostra comercial diferiu-se das demais, e isto ocorreu somente em relação a intensidade de amarelo.

A Tabela 4 apresenta os parâmetros de cor das amostras de manga.

Tabela 4 -Parâmetros de cor das amostras de manga.

\begin{tabular}{cccc}
\hline & $\mathbf{L}^{*}$ & $\mathbf{a}^{*}$ & $\mathbf{b}^{*}$ \\
\hline $\begin{array}{c}\text { in } \\
\text { natura }\end{array}$ & $70,7^{\mathrm{a}} \pm 1,4$ & $10,5^{\mathrm{b}} \pm 1$ & $63,2^{\mathrm{c}} \pm 4$ \\
$\begin{array}{c}\text { Liof. } \\
\text { 48h }\end{array}$ & $65,7^{\mathrm{a}} \pm 2,6$ & $11,8^{\mathrm{b}} \pm 0,4$ & $73,9^{\mathrm{c}} \pm 3,7$ \\
$\begin{array}{c}\text { Liof. } \\
\text { comercial }\end{array}$ & $55,1^{\mathrm{b}} \pm 5$ & $18,2^{\mathrm{c}} \pm 2,1$ & $60,9^{\mathrm{c}} \pm 14$
\end{tabular}

Obs.: médias seguidas pela mesma letra nas colunas não diferem estatisticamente ao nível de 5\% de significância pelo teste de Tukey.

Fonte: Autor (2015).

Observando a Tabela 4 verifica-se que a cor amarela também predomina na manga, como nas demais frutas. Porém, a intensidade da coloração vermelha na manga é maior do que nos outros frutos estudados. Moreira et al. (2013) obtiveram valores de luminosidade e de intensidade de vermelho próximos aos encontrados neste estudo, sendo 65,8 e 9,1, respectivamente. Enquanto que a intensidade de amarelo indicada foi de 40,2; valor inferior ao apresentado na Tabela 4.

Os resultados encontrados por Moreira et al., (2013) para a manga liofilizada apresentaram diferença para os expostos neste trabalho. Este encontrou valores de 52,2 para a luminosidade, -2,01 para a contribuição de verde e 19,2 para a contribuição de amarelo.

Quanto a semelhança estatística dos resultados, foi possível notar que o único parâmetro que apresentou semelhança entre todas as amostras foi a intensidade de amarelo.

\section{CONCLUSÃO}

Através deste estudo foi possível observar que os conteúdos de umidade das frutas in natura foram de $86,8 \%$ para o abacaxi, $71,8 \%$ para a banana e $84,8 \%$ para a manga. As amostras liofilizadas neste trabalho e comercial apresentaram umidade entre 10,8 e $18,6 \%$ para o abacaxi, 10,2 a $11,7 \%$ para a banana e 8,8 a $12,4 \%$ para a manga.

Observou-se que a liofilização é um método eficiente para a prevenção da deterioração da cor das amostras, sendo esta uma das grandes vantagens em relação aos outros métodos de secagem.

Verificou-se a predominância da cor amarela sobre a vermelha, em todas as amostras analisadas. O abacaxi apresentou luminosidade das amostras entre 66, 2 a 76; intensidade de vermelho de $-0,9$ a 4,3 e intensidade de amarelo de 30,3 a 34,2. Ainda, apresentou semelhança estatística para a cor entre todas as amostras, exceto em relação a intensidade de vermelho.

A luminosidade das amostras de banana ficaram entre 71,5 e 72,5; a contribuição de vermelho entre 4,8 e 5,9 e a contribuição de amarelo na faixa de 26,8 a 39,5 . Somente houve divergência estatística nos resultados da intensidade de amarelo das amostras.

Os valores obtidos para os parâmetros de cor das amostras de manga ficaram entre 55,1 a 70,7 para a luminosidade, 10,5 a 18,2 para a intensidade de vermelho e 60,9 e 73,9 para a intensidade de amarelo. O único parâmetro que apresentou semelhança 


\section{ENEMP \\ Th \\ DE SISTEMAS PARTICULADOS \\ São Carlos - SP}

estatística entre todas as amostras foi o da intensidade de amarelo.

\section{REFERÊNCIAS}

ANVISA - Agência Nacional de Vigilância Sanitária. Resolução CNNPA nº 12 de 1978. Regulamento técnico sobre os padrões microbiológicos para alimentos. Acesso em: 12 de Jun. de 2015.

BOBBIO, F.O; BOBBIO, P.A. Introdução à química de alimentos. São Paulo, 1992.

FAO. Tropical fruits commodity notes: Highlights include 2002 production data for mangoes, pineapple, avocado and papaya and a review of preliminary 2002 trade data, 2003.

FRANCIS, F.J. Colorimetry of foods. In: Peleg, M.; Bagley, E.B. Physical properties of foods. Westport:

The

AviPublishingCompany, 1983. p.105-123.

GAVA, A. J. Princípios de Tecnologia de Alimentos. São Paulo,1994.

IBARZ, A.; BARBOSA-CANOVAS, G. V. Deshidratación

OperacionesUnitariasenlaIngeniería de

Alimentos.Lancaster, Basel, 1999.

KROKIDA, M. K. Quality changes during of food materials.Drying Technology in Agriculture and Food Sciences. Mujumdar, Enfield, USA. p. 61-106, 2000.

LIMA, A. Et al. Avaliação das características físico-químicas de bananas desidratadas. In: Congresso Norte Nordeste de Pesquisa e Inovação, 2012, Palmas. Anais do VII CONNEPI. Palmas, Tocantins, 2012. p.1-5.

MOREIRA. Et al.Comportamento das Isotermas de Adsorção do Pó da Polpa de Manga Liofilizada. Revista Brasileira de
Engenharia Agrícola e Ambiental, v.17, p.1093-1098, 2013.

MUJUMDA3R,

A.S.

ResearchandDevelopments in Drying: Recent Trends and Future Prospects. Drying Technology. v.22, p. 1-26, 2004.

OLIVEIRA, M. Efeito da Composição Química, Origem e Grau de Maturação sobre a Cor e a Crocância da Banana Nanica Obtida por Secagem HTST. 2007. 140p. Dissertação (Mestrado em Engenharia de Alimentos) - Universidade Estadual de Campinas. Campinas, São Paulo, 2007.

ORDÓNEZ, J. A. Conceito e objetivos da tecnologia de alimentos. Porto Alegre: Artmed, 2005.p. 13-19.

RAMOS. Et al.Efeito do Tipo de Embalagem e do Tempo de Armazenamento nas Qualidades Físico-Química e Microbiológica de Abacaxi Desidratado. Alimentos e Nutrição,v.19, p.254-269, 2008.

ZOTARELLI, M.F. PORCIUNCULA, B.D.A.; LAURINDO, J.B. A Convective Multi-Flash Drying Process for Producing Dehydrated Crispy Fruits. JournalofFoodEngineering, v.8, p.523-531, 2012.

\section{AGRADECIMENTOS}

À Universidade Federal do Pampa pela bolsa do Programa de Desenvolvimento Acadêmico (PDA). 